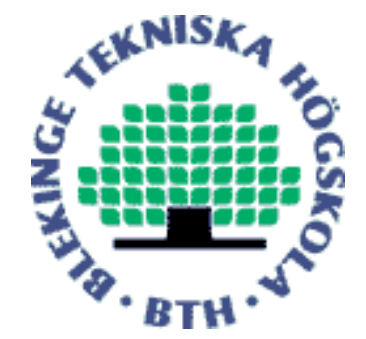

Copyright (C) 2011 IEEE.

Citation for the published paper:

Classification of TCP Connection Termination Behaviors for Mobile Web

Juanid Shaikh, Markus Fiedler, Patrik Arlos, Tahir Minhas, Dennis Collange

Smart Communication Protocols and Algorithms (SCPA)

2011 Houston

This material is posted here with permission of the IEEE. Such permission of the IEEE does not in any way imply IEEE endorsement of any of BTH's products or services Internal or personal use of this material is permitted. However, permission to reprint/republish this material for advertising or promotional purposes or for creating new collective works for resale or redistribution must be obtained from the IEEE by sending a blank email message to pubs-permissions@iee.org.

By choosing to view this document, you agree to all provisions of the copyright laws protecting it. 


\section{Classification of TCP Connection Termination Behaviors for Mobile Web}

\author{
Junaid Shaikh, Markus Fiedler, Patrik Arlos, Tahir Minhas \\ School of Computing \\ Blekinge Institute of Technology \\ Karlskrona, Sweden \\ Email: \{junaid.junaid,markus.fiedler,patrik.arlos,tahir.nawaz.minhas\}@bth.se
}

\author{
Denis Collange \\ Orange Labs \\ Networks and Carriers \\ Sophia Antipolis, France \\ Email: denis.collange@orange-ftgroup.com
}

\begin{abstract}
Many emerging smart applications and services employ Web technology, and users nowadays surf the Web from any device via any kind of access network. Typically, high page latencies trigger users to abort ongoing transfers, resulting in the abrupt terminations of the TCP connections. This paper presents a systematic study of the termination process of the TCP connections and identifies the reasons behind the observed sequences of termination flags. Monitoring and classification of the termination behavior of the TCP connections can provide indications about the user-perceived performance of Web transfers. From the results, it is observed that the TCP termination behavior is heavily-dependent on the client-side application. Therefore, a set of criteria is required to identify the abortions made by the user.
\end{abstract}

\section{INTRODUCTION}

There has been an enormous growth in usage of the Internet on mobile phones during the recent years. According to the study [1], Web usage on mobile phones is expected to represent $50 \%$ of the total Internet usage by 2014. Each mobile device is now equipped with multiple wireless network interfaces, but the performance of these networks is highly varying, mainly due to availability and coverage issues. These performance problems in the wireless networks result in high page latencies and hence affects badly the user experience on the Web. Therefore, the automatic detection of user-perceived performance degradation is an important challenge for network operators. Such a monitoring approach may allow network operators to take user-centric decisions for the switching between multiple wireless technologies when the performance gets poor.

Monitoring of the Transmission Control Protocol (TCP) connection terminations on the Web is one of the ways to monitor user-perceived performance degradation of a service. Normally, users press the Stop or Reload button in the Web browser to abort an on-going transfer when it is much slower than their expectations. These abortions results in early termination of the TCP connections with Reset (RST) flag from the client side. These RST flags can be monitored passively on the network-level to observe the user behavior.

However, some of the studies in the past revealed that there are a significant number of TCP connections on the Internet that consist of one or more TCP RST flags. Client-side applications are the major contributor of these flags [2], [3],
[4]. Some of the client-side Web browsers terminate the TCP connections with a RST flag instead of proper FIN handshake (TCP FIN flags from both sides) which is against the TCP standards mentioned in [5]. But these studies did not explain how to identify the client-side RST flags that result in an early termination of TCP connections (i.e. the RST flags that are sent while the data transfer is still going on).

In [6], the authors presented a criterion to identify the TCP interruptions done by the client. While this criterion is helpful in identifying those TCP connections which are interrupted, it does not guarantee whether interruptions are due to the abortion made by the user or automatically by the Web browser. It stimulates the need for an improved criterion that identifies the interruptions done by the user and also gives an idea about the type of user action performed in the Web browser.

In this work, we have performed a systematic study to show in detail the sequence of termination flags, in order to identify the transfers aborted by the users. Sequence of these termination flags occurred as a result of different actions as listed in Table I, performed in the Web browser. Hence, monitoring and classification of the termination type in real scenario may provide rough indications whether a connection was interrupted by the user or not. To ensure a fair and representative comparison, we have conducted a number of controlled experiments with various Web browsers. These experiments are done on smartphones to test the mobile Web browsers. Network operators and Web service providers can use this knowledge to passively monitor the behavior of users over time, and manage their resources accordingly to guarantee a quality user experience. The research community working on the network Quality of Experience can use this study to validate it against the subjective experiments with real users. Finally, Web users can also use results to choose Web browsers that are operating according to the rules defined by the standards.

The remainder of this paper is organized as follows. Section II describes the methodology used to conduct this study and Section III presents the experimental setup. Section IV discusses the sequence of termination flags observed with different Web browsers. Section V presents the timing behavior of the termination process. Section VI concludes this study 
and finally, Section VII discusses about the extension of this work in the future.

\section{Methodology}

We conducted a set of active tests to observe the sequence of TCP termination flags exchanged in both directions. To execute the tests, we established an isolated environment. These tests were conducted by accessing a Webpage on a smartphone. The Webpage was located on a local Web server.

Two types of tests were performed: Uninterrupted and interrupted. In the uninterrupted tests, the user issues a Webpage request and then allows the transfer of the Webpage to finish completely. In the interrupted test, user aborts an ongoing transfer of Webpage by performing some action in the Web browser. The user action could be either pressing the Stop or the Reload button, exiting the Web browser or clicking a hyperlink on the Webpage. These actions are further mentioned in Table I.

In order to study the impact of the content type, three Webpages were developed. One Webpage had simple text, the second one had an image and the third one had a flash video, played in a shockwave player on the Webpage. Since the results of the tests with text and image Webpages were almost similar to each other, we only present the results related to text and to video in the rest of this paper.

Moreover, tests were performed on three popular mobile platforms: Windows 6.5 (HTC HD2), Android 2.2 (HTC Desire HD) and Symbian 3.0 (Nokia N8). Built-in Web browsers were used on each of these platforms as external browsers were not supporting the video content. The Web browser used by Windows 6.5 is Microsoft Internet Explorer 6.0. The user agent string in the HTML header reports Android's Web browser as Mobile Safari and Symbian's Web browser as Browser NG which is used on the Nokia mobile phones. On Android and Symbian platforms, the built-in Web browsers use Webkit as the HTML rendering engine which is an open-source Web browser engine [7].

Summarizing the above description, each experiment was differentiated from other based on either the user action, content type or mobile platform. Hence, a full factorial experimental design with $4 \times 2 \times 3=24$ experiments were established. Each experiment was repeated 40 times that made total number of runs to be $24 \times 40=960$.

TABLE I

DIFFERENT TYPES OF USER ACTIONS

\begin{tabular}{|l|c|}
\hline Type & Description \\
\hline Uninterrupted & User allows the page to load completely \\
\hline Kill-browser & $\begin{array}{r}\text { User kills the browser before the } \\
\text { page is completely loaded }\end{array}$ \\
\hline Stop/Reload & $\begin{array}{c}\text { User presses the stop or reload button before the } \\
\text { page is completely loaded }\end{array}$ \\
\hline Link-follow & $\begin{array}{c}\text { User clicks another link before the } \\
\text { page is completely loaded }\end{array}$ \\
\hline
\end{tabular}

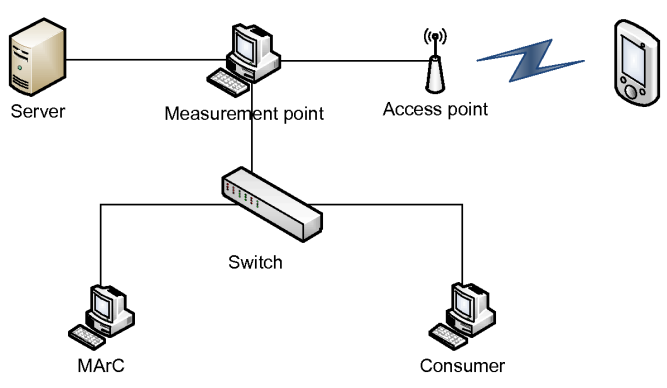

Fig. 1. Setup

\section{TEST-BED SETUP}

The setup used for experiments is based on Distributed Passive Measurement Infrastructure (DPMI) [8], as shown in Figure 1. For capturing of traffic traces, a Measurement Point (MP) equipped with two Data Acquisition and Generation (DAG) cards was used [9]. It captures traffic in both directions. The time of MP is synchronized with Network Time Protocol (NTP) [10] and an input pulse of GPS is used for the clock ticks. The network, transport and application headers are captured and then forwarded to the Consumer, where they are stored for further analysis. A Measurement Area Controller (MArC) controls the MP and the Consumer. Apache 2.2 is chosen as Webserver in these experiments as it is the most popular Webserver in use [11]. The mobile client in the Figure 1 is a smartphone that is used for requesting the Webpage by user via the access point using IEEE $802.11 \mathrm{~b}$ WiFi technology. A full duplex link of $100 \mathrm{Mbps}$ is used between Web server and access point. The TCP version used by the Web server is TCP New Reno.

\section{TERMINATION FLAGS}

Table II summarizes the different termination types seen from all the experiments. Termination type here refers to the sequence of terminating flags that were seen at the end of a TCP connection. Five different types of terminations are observed. Sequence of these termination flags occurred as a result of different actions as listed in Table I, performed in the Web browser.

TABLE II

DifFEREnt SEQuences of Termination Flags Observed

\begin{tabular}{|l|c|}
\hline Termination & Description \\
\hline$F_{s} F_{c} R_{c}$ & $\begin{array}{c}\text { A FIN from the server followed by a FIN } \\
\text { and then one or more RSTs from the client }\end{array}$ \\
\hline$R_{c} F_{c} R_{s}$ & $\begin{array}{c}\text { One or more RSTs from the client followed } \\
\text { by a FIN from the client and a RST from the server }\end{array}$ \\
\hline$F_{s} F_{c}$ & A FIN from the server followed by a FIN the client \\
\hline$F_{c} R_{c}$ & $\begin{array}{c}\text { A FIN from the client followed by one or } \\
\text { more RSTs from the client }\end{array}$ \\
\hline$R_{c}$ & One or more RSTs from the client \\
\hline
\end{tabular}




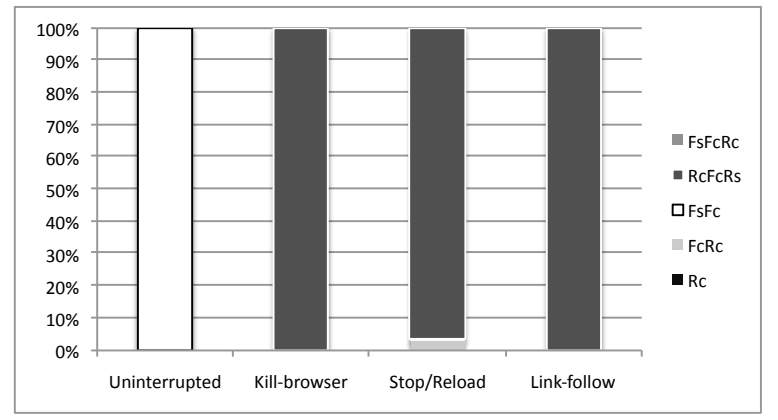

Fig. 2. Termination flags with text Webpage on Symbian

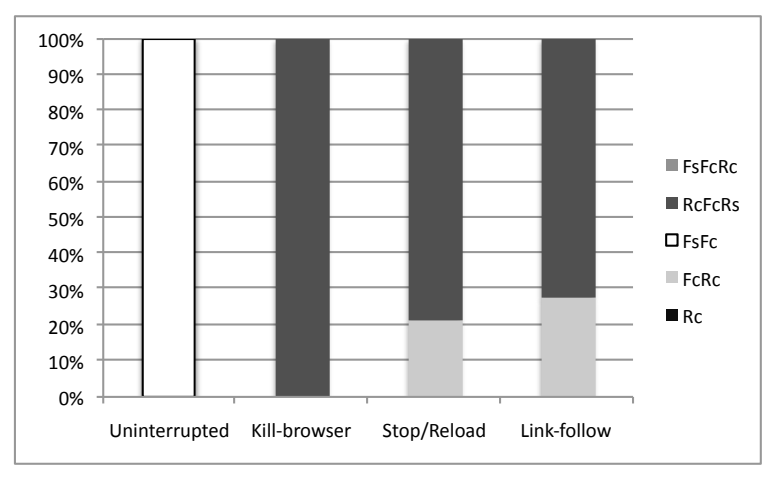

Fig. 3. Termination flags with video Webpage on Symbian

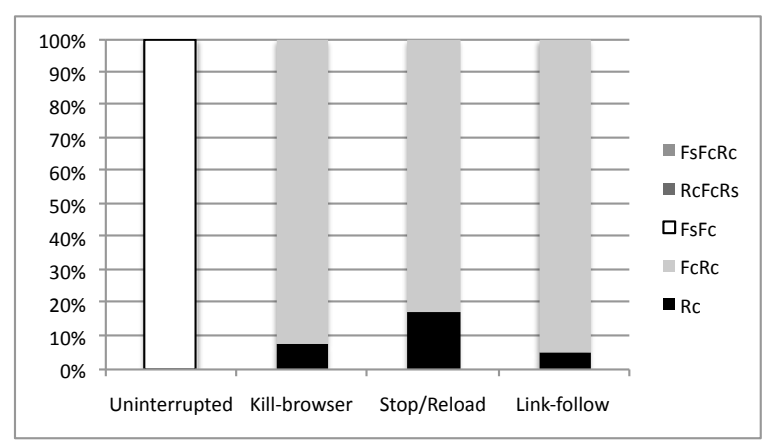

Fig. 4. Termination flags with text Webpage on Android

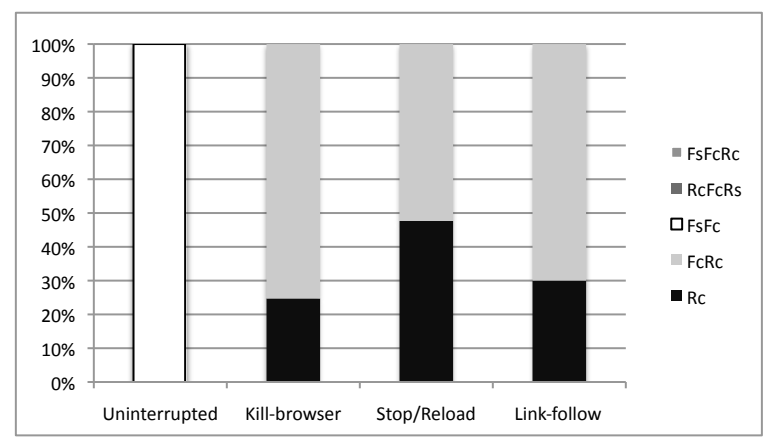

Fig. 5. Termination flags with video Webpage on Android

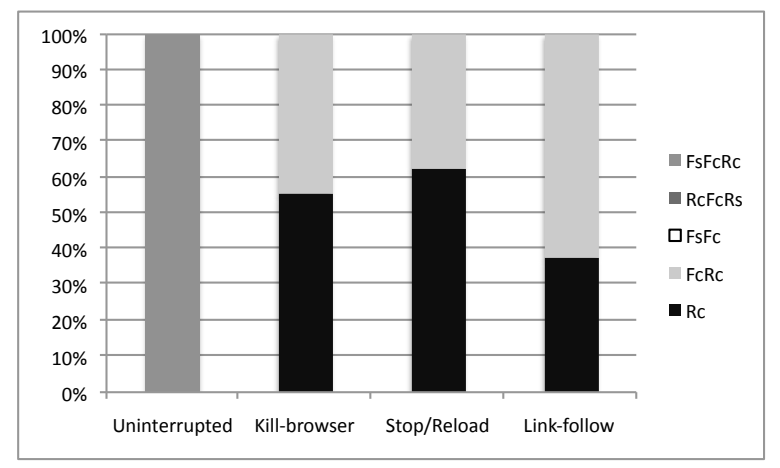

Fig. 6. Termination flags with text Webpage on Windows

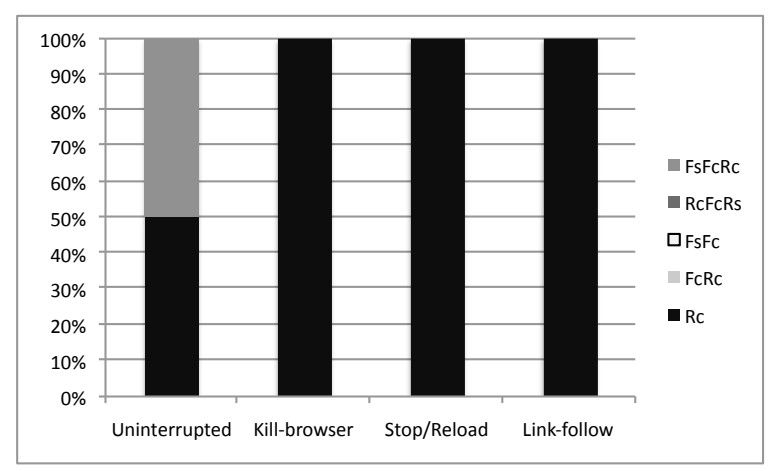

Fig. 7. Termination flags with video Webpage on Windows

\section{A. Uninterrupted transfers}

The bar charts in Figures 2-7 highlight the number of each terminating sequence observed as a consequence of different user actions performed in different Web browsers. On Symbian and Android platforms, all the TCP connections ended with a proper FIN handshake. After the data transfer, a FIN from the server is sent which is followed by a FIN from the client-side to end the connection. This type of termination follows the rules as described by the standards [5].

Client-side RST flag On the Windows platform however, the text-based Webpage transfer is finished with a FIN from server, followed by a FIN and then a RST flag from the client. This RST flag appears to be a reaction of the client to the ACK received from the server, which triggers the client to immediately shutdown the connection by sending a RST flag. This behavior is found to be consistent in all the transfers.

Multiple TCP connections per transfer: Subsequently, when the video-based Webpage is downloaded from the Windows platform, there is another interesting pattern seen in the connection termination process, as shown in Figure 8. After receiving the base file, the client makes a GET request for the video player. It then immediately terminates the connection with a RST flag and initiates the new connection with a SYN handshake. The GET request for the previous file is thus repeated once again and then the video is played in the Web browser. The second connection is terminated similarly as was observed in the case of text-based Webpage. Hence, two connections are opened for playing video in the Web 


\begin{tabular}{|c|c|c|}
\hline \multicolumn{3}{|c|}{ Port Client } \\
\hline 1343 & SYN & \\
\hline 1343 & SYN, ACK & \\
\hline 1343 & GET index.html & \\
\hline 1343 & HTTP/1.1200 OK & \\
\hline 1343 & GET jww.swf & \\
\hline 1343 & RST & \\
\hline 1344 & SYN & \\
\hline 1344 & SYN, ACK & \\
\hline 1344 & GET jww.swf & \\
\hline 1344 & HTTP/1.1200 OK & \\
\hline 1344 & GET nature.flv & \\
\hline 1344 & HTTP/1.1200 OK & \\
\hline 1344 & FIN & \\
\hline 1344 & FIN & \\
\hline 1344 & RST & 8 \\
\hline
\end{tabular}

Fig. 8. Video transfer on the Windows platform

browser. The connection establishment procedure creates extra overhead which affects badly the overall speed of the transfer. The TCP connection also goes into the slow start phase once again. The client-side software should avoid this kind of behavior as the opening of multiple TCP connections per transfer may degrade the performance of the transfer.

\section{B. Interrupted transfers}

Interrupted transfers are those in which a user aborts an on-going transfer by manually performing either of the three actions (before the end of the download) in Web browser: Pressing the stop or reload button, exiting the browser or clicking a hyperlink on the Webpage. The results in Figures 2-7 illustrate that the connection termination pattern is similar when the interruptions are made from Android and Windows platform, while it is slightly different in the case of the Symbian platform.

Server-side RST flag: While using the Symbian platform, a large ratio of TCP connections were terminated with one or more RST flags from the client, followed by a FIN flag from the client and then a RST flag from server. The reason why the server responded with a RST flag is that when it received a RST flag from client, it assumed the connection was already closed and therefore, when it received an additional packet from client (containing a FIN flag) on the same port, it responded with a RST flag to once again signal the end of the connection.

Retransmissions: In a few interrupted transfers on Symbian and Windows, and in the majority of transfers on Android, connections were terminated with a FIN flag followed by one or more RST flags from the client. By looking at the interrupted traffic traces, we found out that, when the client starts termination process with a FIN flag, then the server responds with the retransmission of previous unacknowledged segments. On receiving the retransmitted segments, the client tears down the connection by sending one or more RST flags. This kind of anomaly may result in the wrong estimation
TABLE III

STATISTICS OF TIME-TO-TERMINATION

\begin{tabular}{|l|c|c|c|c|}
\hline \multirow{2}{*}{ Platform/Content } & \multicolumn{2}{|c|}{ Uninterrupted } & \multicolumn{2}{|c|}{ Interrupted } \\
& $\bar{t}_{\mathrm{t}}$ & $\sigma_{\mathrm{t}}$ & $\bar{t}_{\mathrm{t}}$ & $\sigma_{\mathrm{t}}$ \\
\hline Android/Text & $5.52 \mathrm{~s}$ & $0.004 \mathrm{~s}$ & $0.10 \mathrm{~s}$ & $0.16 \mathrm{~s}$ \\
\hline Android/Video & $5.52 \mathrm{~s}$ & $0.004 \mathrm{~s}$ & $0.01 \mathrm{~s}$ & $0.002 \mathrm{~s}$ \\
\hline Symbian/Text & $5.65 \mathrm{~s}$ & $0.09 \mathrm{~s}$ & $0.78 \mathrm{~s}$ & $0.36 \mathrm{~s}$ \\
\hline Symbian/Video & $5.52 \mathrm{~s}$ & $0.03 \mathrm{~s}$ & $0.01 \mathrm{~s}$ & $0.02 \mathrm{~s}$ \\
\hline Windows/Text & $5.58 \mathrm{~s}$ & $0.04 \mathrm{~s}$ & $0.19 \mathrm{~s}$ & $0.02 \mathrm{~s}$ \\
\hline Windows/Video & $0.01 \mathrm{~s}$ & $0.02 \mathrm{~s}$ & $0.01 \mathrm{~s}$ & $0.003 \mathrm{~s}$ \\
\hline
\end{tabular}

of loss rates on the network. On the Windows platform, the majority of the connections were terminated with one or more RST flags from the client without any FIN flag.

Indication of user-interrupted transfers: Generally, from all the above observations of interrupted connections, one thing is common that at least one RST flag from the client-side is seen regardless of the platform. Another important evidence about the user-generated interruption is that more than one consecutive RST flags was seen in most of the cases as soon as the user performs an interruption in the Web browser.

Indication of non-user-interrupted transfers: On the other hand, a single RST flag is seen if the transfer is not interrupted by user on which the RST flag is sent automatically by the client-side software.

\section{TIME-TO-TERMINATION}

To further understand the difference between userinterrupted and client-side application-interrupted connections, we present the time-to-termination $t_{\mathrm{t}}$, which refers to the time elapsed from the last data packet from server $t_{\mathrm{s}}$ to the first termination flag $t_{\mathrm{f}}$ observed:

$$
t_{\mathrm{t}}=t_{\mathrm{f}}-t_{\mathrm{s}}
$$

Table III lists the mean time-to-termination $\left(\bar{t}_{\mathrm{t}}\right)$ and standard deviation $\left(\sigma_{\mathrm{t}}\right)$ observed from our traces for interrupted and uninterrupted experiments. The mean time-to-termination $\bar{t}_{\mathrm{t}}$ is taken by averaging the time-to-terminations $t_{\mathrm{t}}$ of 40 repetitions of each experiment, based on which the standard deviation $\sigma_{\mathrm{t}}$ of an experiment is calculated.

In the case of uninterrupted connections, $\bar{t}_{\mathrm{t}}$ is around $5.5 \mathrm{~s}$. These terminations mainly occurred as a result of FIN flag from the server. It shows the time-out value of the Apache Web server that closes a persistent connection roughly after $5 \mathrm{~s}$ of idle time. When the video-based Webpage is requested on the Windows platform, $\bar{t}_{\mathrm{t}}$ reduces to $0.01 \mathrm{~s}$. This is because two connections are opened when the video-based Webpage is called. The first connection terminates with $\bar{t}_{\mathrm{t}}$ of $0.01 \mathrm{~s}$ and the next connection with $\bar{t}_{\mathrm{t}}$ of around $5.5 \mathrm{~s}$. Hence, in this case it becomes difficult to differentiate between user-interrupted and client-side application-interrupted connections based on the time-to-termination.

For interrupted connections, we observe a notable difference between the values of time-to-termination due to the requested content type. When text pages are requested, the first terminating flag is seen $0.1-0.8 \mathrm{~s}$ after the last data packet from 
the server. In contrast, these values are reduced to $0.01 \mathrm{~s}$, when video pages are requested. This reduction in $\bar{t}_{\mathrm{t}}$ is because the inter-arrival time between packets is smaller than the ones observed in the case of text page and therefore, more packets are in flight when the user interrupts the transfer.

The value of standard deviation in most of the cases is very small i.e. below $100 \mathrm{~ms}$. However, it gets higher when the Web pages containing the text are aborted on the Android and the Symbian platforms. It is because the download time in the case of text pages is very small (i.e. almost around $1 \mathrm{~s})$ therefore, the time required for the user to interrupt the transfer is also very small. Sometimes the page is interrupted at the middle of the transfer (when many data packets are in flight), and sometimes it is interrupted at the tail of the transfer (when not many packets are in flight). Due to this varying user behavior, we observe the higher standard deviation in the time-to-terminations.

\section{CONCLUSION}

In this paper, we studied the TCP connection termination process resulting from different user actions in various Web browsers. We found out that the connection termination behavior is heavily dependent on the type of Web browser used. Therefore, the TCP RST flag alone, cannot be used to detect the user action performed in the Web browser. The results presented in this study provide a baseline for the development of a set of criteria for monitoring user-perceived performance of networks.

Along the way, we also deduct from our observations that a better management approach is needed between client-side Web browsers and servers to improve the performance of TCP connections. Hence, the client-side implementation of TCP connections according to the standards and close cooperation between Web browsers and servers can be helpful in raising the Web browsing experience of users.

\section{FUTURE WORK}

Our short-term future work includes the study of the TCP termination process with other popular Web browsers. We will then propose a set of criteria for the passive monitoring of the user-perceived performance of Web transfers. Tests will subsequently be conducted with real users for the validation of the criteria. Finally, the criteria will be used to model the network performance as perceived by the users.

\section{ACKNOWLEDGMENT}

This work has been supported by the FP7 Network of Excellence Euro-NF (contract number 216366). We would like to thank Prasanna Amburu, Anil Verma, Anil Kumar and Dileesh Kunpuru for their continuous help in conducting these experiments.

\section{REFERENCES}

[1] http://www.itu.int/ITU-D/ict/newslog/Mobile+Internet+Users +To+Top+ 17+Billion+By+2013.aspx, Last visited: July 14, 2011.

[2] M. Arlitt, C. Williamson, An Analysis of TCP Reset Behaviour on the Internet, ACM SIGCOMM Computer Communication Review, Volume 35, Number 1, January 2005.
[3] W. John, S. Tafvelin, T. Olovsson, Trends and Differences in ConnectionBehavior within Classes of Internet Backbone Traffic, PAM 2008, LNCS 4979, pp. 192-201, 2008.

[4] K. Fukuda, An Analysis of TCP Passive Measurements, $\quad$ TMA 2011, LNCS 6613, pp. 29-36, 2011.

[5] J. Postel Transmission Control Protocol, RFC 793, 1981.

[6] D. Rossi, M. Mellia, C. Casetti, User Patience and the Web: A hands-on investigation, IEEE Globecom, San Francisco USA, December 2003

[7] http://www.webkit.org, Last visited: August 29, 2011.

[8] P. Arlos, M. Fiedler, A. Nilsson, A Distributed Passive Measurement Infrastructure, Lecture Notes in Computer Science, Volume 3431/2005, 2005.

[9] http://www.endace.com/endace-dag-high-speed-packet-capturecards.html, Last visited: July 13, 2011.

[10] http://www.ntp.org/, Last visited: July 14, 2011.

[11] http://news.netcraft.com/archives/2011/05/02/may-2011-web-serversurvey.html, Last visited: July 15, 2011. 